\title{
Tumor microenvironment related novel signature predict lung adenocarcinoma survival
}

\author{
Juan Chen ${ }^{1}$, Rui Zhou ${ }^{\text {Corresp. } 1}$ \\ ${ }^{1}$ Respiratory Medicine, The Second Xiangya Hospital of Central South University, Changsha, Hunan, China \\ Corresponding Author: Rui Zhou \\ Email address: zhourui2355@csu.edu.cn
}

Background:Lung adenocarcinoma (LUAD) is the most common histological type of lung cancers, which is the primary cause of cancer-related mortality worldwide. Growing evidence has suggested that tumor microenvironment (TME) plays a pivotal role in tumorigenesis and progression. Hence, we investigate the correlation of TME related genes with LUAD prognosis.

Method:The information of LUAD gene expression data was obtained from The Cancer Genome Atla(TCGA). According to their immune/stromal scores calculated by the ESTIMATE algorithm, differentially expressed genes(DEGs) were identified. Then, we performed univariate Cox regression analysis on DEGs to obtain genes that are apparently bound up with LUAD survival (SurGenes). Functional annotation and protein-protein interaction (PPI) was also conducted on SurGenes. By validating the SurGenes with another data sets of lung cancer from Gene Expression Omnibus (GEO), 106 TME related SurGenes were generated. Further, intersection analysis was executed between the 106 TME related SurGenes and hub genes from PPI network, PTPRC and CD19 were obtained. And Gene Set Enrichment Analysis and CIBERSORT analysis were performed on PTPRC and CD19. Based on the TCGA LUAD dataset, we conducted factor analysis and Step-wise multivariate Cox regression analysis for 106 TME related SurGenes to construct the prognostic model for LUAD survival prediction. LUAD dataset in GEO (GSE68465) was used as the testing dataset to confirm the prognostic model. Multivariate Cox regression analysis was used between risk score from the prognostic model and clinical parameters.

Result:106 TME related genes were collected in our research totally, which were markablely correlated with the overall survival(OS) of LUAD patient. Bioinformatics analysis suggest them mainly concentrated on immune response, cell adhesion, and extracellular matrix. More importantly, among 106 TME related SurGenes, PTPRC and CD19 were highly interconnected nodes among PPI network and correlated with immune activity, exhibiting significant prognostic potential. The prognostic model was a weighted linear combination of the 106 genes, by which the low-OS LUAD samples could be separated from the high-OS samples with success. This model was also able to rebustly predict the situation of survival (training set: $p$-value $<0.0001$, area under the curve $(A U C)=0.649$; testing set: $p$-value $=0.0009, A U C=0.617$ ). By combining with clinical parameters, the prognostic model was optimized. The AUC achieved 0.716 for 3 year and 0.699 for 5 year.

Conclusion:A series of TME-related prognostic genes were acquired in this research, which could reflect immune disorders within tumor microenvironment, and PTPRC and CD19 show the potential to be an indicator for LUAD prognosis and tumor microenvironment modulation. The prognostic model constructed base on those prognostic genes presented a high predictive ability, and may have clinical implications in the overall survival prediction of LUAD. 


\section{Tumor microenvironment related novel signature 2 predict lung adenocarcinoma survival}

3 Juan Chen ${ }^{1}$

$4{ }^{1}$ Respiratory Medicine, The Second Xiangya Hospital of Central South University. Changsha, 5 Hunan, China

7 Corresponding Author:

8 Rui Zhou ${ }^{1}$

9 No.139 Middle Renmin Road, Changsha, Hunan, 410011, China.

10 Email address: zhourui2355@csu.edu.cn

\section{Abstract}

Background: Lung adenocarcinoma (LUAD) is the most common histological type of lung cancers, which is the primary cause of cancer-related mortality worldwide. Growing evidence has suggested that tumor microenvironment (TME) plays a pivotal role in tumorigenesis and progression. Hence, we investigate the correlation of TME related genes with LUAD prognosis.

Methods: The information of LUAD gene expression data was obtained from The Cancer Genome Atlas (TCGA). According to their immune/stromal score calculated by the ESTIMATE algorithm, differentially expressed genes (DEGs) were identified. Then, we performed univariate Cox regression analysis on DEGs to obtain genes that are apparently bound up with LUAD survival (SurGenes). Functional annotation and protein-protein interaction (PPI) was also conducted on SurGenes. By validating the SurGenes with another data sets of lung cancer from Gene Expression Omnibus (GEO), 106 TME related SurGenes were generated. Further, intersection analysis was executed between the 106 TME related SurGenes and hub genes from PPI network, and PTPRC and CD19 were obtained. And Gene Set Enrichment Analysis (GSEA) and CIBERSORT analysis were performed on PTPRC and CD19. Based on the TCGA LUAD dataset, we conducted factor analysis and Step-wise multivariate Cox regression analysis for 106 TME related SurGenes to construct the prognostic model for LUAD survival prediction. LUAD dataset in GEO (GSE68465) was used as the testing dataset to confirm the prognostic model. Multivariate Cox regression analysis was used between risk score from the prognostic model and clinical parameters. 
32 Results: $106 \mathrm{TME}$ related genes were collected in our research totally, which were markablely 33 correlated with the overall survival (OS) of LUAD patient. Bioinformatics analysis suggest them 34 mainly concentrated on immune response, cell adhesion, and extracellular matrix. More 35 importantly, among 106 TME related SurGenes, PTPRC and CD19 were highly interconnected 36 nodes among PPI network and correlated with immune activity, exhibiting significant prognostic 37 potential. The prognostic model was a weighted linear combination of the 106 genes, by which 38 the low-OS LUAD samples could be separated from the high-OS samples with success. This 39 model was also able to rebustly predict the situation of survival (training set: $p$ value $<0.0001$, 40 area under the curve (AUC) $=0.649$; testing set: $p$-value $=0.0009$, area under the curve (AUC) $41=0.617)$. By combining with clinical parameters, the prognostic model was optimized. The AUC achieved 0.716 for 3 year and 0.699 for 5 year.

Conclusion: A series of TME-related prognostic genes were acquired in this research, which could reflect immune disorders within tumor microenvironment, and PTPRC and CD19 show the potential to be an indicator for LUAD prognosis and tumor microenvironment modulation. The prognostic model constructed base on those prognostic genes presented a high predictive ability, and may have clinical implications in the overall survival prediction of LUAD.

\section{Introduction}

50

51

52

53

54

55

56

57

58

59

60

61

62

63

64

65

66

67

68

Lung cancer is the most common cause of cancer-related deaths worldwide. ${ }^{1}$ Non-small cell lung cancer (NSCLC) represents $85 \%$ of lung cancers, mainly including lung adenocarcinoma (LUAD), lung squamous cell carcinoma (LSCC). ${ }^{2}$ Notably, the incidence of LUAD significantly increased and surpassed of LSCC and it constitutes nearly $40 \%$ of all lung malignancies. ${ }^{3}$

Although with multiple kinds treatment methods, including surgery, chemotherapy, radiotherapy, and dramatic treatment shift of some targeted therapeutic agents, the prognosis for LUAD patients remains poor worldwide, with 5 -year relative survival currently at $18 \% .^{4-6}$ Therefore, understanding the mechanism of carcinogenesis and therapeutics of lung cancer is quietly important.

At present, the pathogenesis of LUAD has not been adequately described, meanwhile, there are increasing study support the view of tumor microenvironment critically influence gene expression of tumor tissues, and the clinical outcomes further. The tumor microenvironment (TME) is a complicated mixture, immune and stromal cells are two major types of non-tumor factors. They have been certified to promote the development of diagnostic and prognostic assessment process of lung cancer ${ }^{7-9}$. Hence, exploring the molecular composition and function of the TME is critical to effectively manage cancer progression and immune response ${ }^{10-11}$.

Previous studies have solved the problem of the complexity of tumor infiltrating immune cells in LUAD. Most of these studies assessed tumor-infiltrating immune cells by immunohistochemical analysis of a single marker. ${ }^{14-16}$ Fortunately, computational analysis of tumor immune cell 
69

70

71

72

73

74

75

76

77

78

79

80

81

82

83

84

85

86

87

88

89

90

91

92

93

94

95

96

97

98

99

100

101

102

103

104

interactions is now available by bioinformatics tools. In 2013, Yoshihara firstly reported an algorithm called ESTIMATE (Estimation of STromal and Immune cells in MAlignant Tumor tissues using Expression data). The infiltration level of stromal and immune cells was predicted by calculating stromal and immune score based on data from the Cancer Genome Atlas (TCGA) data sets by ESTIMATE. ${ }^{17}$ Subsequent reports applied the innovative algorithm to different cancer, such as prostate cancer, ${ }^{18}$ breast cancer, ${ }^{19}$ and colon cancer, ${ }^{20}$ which further confirmed the effectiveness of the algorithm based on big database.

We analyzed immune/stromal score of LUAD cohorts from TCGA, which was derived from ESTIMATE algorithm, and extracted a series of TME associated prognostic gene in LUAD. Among those TME associated prognostic gene, PTPRC and CD19 were particular interested for they are highly interconnected in PPI network, and closely correlated with immune-related activity by GSEA analysis and CIBERSORT analysis. Combine those prognostic genes, we constructed a prognostic model which could provide a moderate OS prediction for LUAD alone, and provide a robust prediction with clinical parameters.

\section{Materials \& Methods}

\section{Data acquisition}

From TCGA database, including 576 samples, gene expression RNAseq and clinical information such as pathological stage, survival of LUAD were downloaded and prepared for the analysis of differential expression. The data set was submitted by University of North Carolina TCGA genome characterization center based on the Illumina HiSeq 2000 RNA Sequencing platform (Oct 13, 2017). We downloaded the estimate, immune and stromal score of 517 LUAD samples from TCGA in ESTIMATE website. Stromal score captures the presence of stroma in tumor tissue, immune score represents the infiltration of immune cells in tumor tissue, and estimate score that infers tumor purity. The estimate score is equal to the immune score plus the stromal score. Fifty-nine samples were deleted for lack of immune and stromal score in ESTIMATE website. From GEO database with the accession number GSE68465, consisting of 442 samples, gene expression profile and LUAD clinical covariates (stage, age, sex) were obtained. ${ }^{21}$ After using the Robust Multichip Averaging (RMA) method to normalize the sequence matrix data, the data was used to validate the differential expression gene and test the prognostic model. The data set was submitted by Mervi Heiskanen based on Affymetrix HG-U133A (May 01, 2015). ${ }^{22}$ From GEO database with the accession number GSE14814, including 133 non-small cell lung cancer samples, was downloaded to compared the prognostic model with other gene expression signature. 
105 Distinguishing of differentially expressed genes (DEGs)

106 The raw data of TCGA was pre-processed by limma algorithm. ${ }^{23}$ The adjusted P-values (adj. p) $107<0.05$ and $|\log 2(\mathrm{FC})|>1$ were set as the cut-offs to screen for differentially expressed genes.

108

109 Heatmaps and clustering analysis

110 ClustVis web tool was used to create heatmaps and clustering.24

112 Enrichment analysis of DEGs.

113 DAVID conducted functional enrichment analysis, including biological process, molecular 114 function and cell component to analyze $\mathrm{DEGs},{ }^{25}$ an essential foundation for visualization, 115 annotation, and integrated discovery. KEGG (Kyoto Encyclopedia of Genes and Genomes) 116 pathways was also performed by the database of DAVID. Whole genome was the background 117 using DAVID. False discovery rate $($ FDR $)<0.05$ was used as the cut-off.

118

119

120

121

122

123

124

125

126

127

128

129

130

131

132

133

134

135

136

137

138

\section{Screening of survival-related DEGs}

Kaplan-Meier plots and univariate Cox regression were used to visualize the association between the genes expression and overall survival of patients to explore its prognostic value. Statistical significance was examined using the Log-rank test. $\mathrm{P}<0.05$ were considered statistically significant.

\section{Integration of PPI network and screening of modules}

Search Tool for the Retrieval of Interacting Genes (STRING) online database was used to retrieve predicted PPIs. Only interactions with a combined score $>0.4$ of all associations obtained in STRING were selected to construct the PPI network using Cytoscape software. ${ }^{26}$ The Molecular Complex Detection (MCODE) plugin in Cytoscape was then utilized to find clusters of the PPI network. Top 8 nodes ranked by degree were identified as hub genes.

\section{Gene Set Enrichment Analysis}

From Molecular Signatures Database, Hallmark and C7 gene sets v6.2 collections were downloaded as the target sets. GSEA performed using the software gsea-3.0. 517 samples from TCGA were used for GSEA, and only gene sets with nominal (NOM) p-value $<0.05$ and FDR $<$ 0.25 were considered as significant.

\section{TICs Profile}


139 CIBERSORT computational method was applied for estimating the tumor-infiltrating immune

140

141

142

143

144

145

146

147

148

149

150

151

152

153

154

155

156

157

158

159

160

161

162

163

164

165

166

167

168

169

170

171

subsets (TICs) abundance profile in all tumor samples, which followed by quality fifiltering that only 517 tumor samples with $\mathrm{p}<0.05$ were selected for the following analysis.

\section{Construction of the risk assessment model}

To reduce the dimensionality and eliminate collinearity, factor analysis was performed on 106 prognostic genes. Then we used the Step-wise multivariate Cox regression analysis to obtain factors correlated with overall survival significantly (SurFactors). By combining the score coefficient of factors weighted by their regression coefficients and standard deviation, the risk index of each gene was calculated as follows:

$$
\alpha_{i}=\frac{\sum_{j=1}^{m} \beta_{j} \times f a c_{i j}}{s t d_{i}}
$$

where $\alpha_{i}$ was the risk index of gene $i, m$ the number of SurFactors, $\beta_{j}$ the regression coefficient of factor $j$ in multivariate Cox regression analysis, $f a c_{i j}$ the factor score coefficient of $j$-th factor over gene $i$ and $s t d_{i}$ the standard deviation of gene $i$.

By combining the expression values of prognostic genes weighted by their risk index, the following risk scores can be established for each patient:

$$
\text { Risk_score }=\sum_{i=1}^{n} \exp _{i} * \alpha_{i}
$$

where $n$ was the number of prognostic genes, and $\exp _{i}$ the expression value of gene $i$.

\section{Survival and statistical analysis}

We calculated risk score (RS) for every sample from testing data set based on the prognostic model and divided the specimens into two different groups based on the median RS. KaplanMeier survival curves were drawn and compared the two subgroups via log-rank tests. We divided the samples into two group according to their overall survival, receiver operating characteristic (ROC) curves were drawn by IBM SPSS statistics 22, and area under the curve (AUC) was calculated. Moreover, the univariate and multivariate analyses of survival were conducted to identify the prognostic factors for LUAD patients from TCGA data set. A nomogram and Calibration plots were established based on the TCGA LUAD cohort. The nomogram and calibration plot analysis were conducted by using the R package "rms" and "rmda." All tests were two-tailed, and $\mathrm{P}<0.5$ was considered statistically significant. All statistical analyses were conducted using R software version 3.4.2.

\section{Results}




\section{ESTIMATE algorithm of LUAD}

173 Transcriptional expression profiles and clinical information of 517 LUAD patients were

174 collected from TCGA database. Among them, 277 (53.6\%) patients were female, 240 (46.4\%)

175 were male. Pathological stage included 277 patients (53.6\%) of stage I , $122(23.6 \%)$ stage II ,84

176 (16.3\%)stage III ,26 (5\%) stage IV and $8(1.5 \%)$ unknown. Based on ESTIMATE algorithm, the 177 scores of stromal and immune were calculated, ranging from-1355.85 to 3286.67 and -1959.31

178 to2098.77, respectively. The Estimate score was significantly associated with Pathological stage

179 (Figure 1A, $\mathrm{p}=0.0436$ ). The lowest Estimate score was at the most advanced pathological stage,

180 the stage IV. Immune score showed remarkable prognostic potential, correlated with the

181 pathological stage (Figure $1 \mathrm{~B}, \mathrm{p}=0.026$ ), while stromal score showed no correlation with the

182 pathological stage (Figure 1C, $\mathrm{p}=0.145$ ).

183

184 To explore whether the potential correlation existing between survival benefits and 185 immune/stromal score, 517 LUAD patients were divided into high and low score groups based

186 on their scores. Kaplan-Meier survival curves (Figure 1D) showed that immune score

187 significantly correlated with overall survival. Compared to the cases in the low score group, 188 patients with high immune score have longer median overall survival ( $1725 \mathrm{~d}$ vs. $1235 \mathrm{~d}, \mathrm{p}=$ 1890.0152 in log-rank test). Although there was no statistically significant correlation between the 190 stromal score and overall survival, patients with higher stromal score had longer median overall 191 survival (Figure 1E, $1830 \mathrm{~d}$ vs. $1293 \mathrm{~d}, \mathrm{p}=0.0599$ in log-rank test).

192

193

194

195

196

197

198

199

200

201

202

203

204

205

206

\section{Identification of DEGs}

To identify DEGs profiles according to immune/stromal score, we obtained the gene expression array of 517 LUAD patients obtained TCGA database. Based on the comparison of immune score of high group and low group, after analysis with the limma software package algorithm, there were 903 genes up-regulated and 56 genes down-regulated. We draw the heatmap with the top highly variate gene to (Figure 2A). Similarly, 1007 up-regulated genes and 30 down regulated genes were obtained for high stromal score compared with low score (Figure 2B). It is obvious that up-regulated DEGs, no matter for comparison based on immune score nor stromal score, take the major part in total DEGs. Therefore, we decide to focus on up-regulated DEGs in further analysis.

\section{Enrichment analysis of DEGs}

To reveal the biological function of the DEGs, GO and KEGG pathway enrichment analysis were performed for the up-regulated DEGs. GO analysis showed that immune up-regulated 
207 DEGs were remarkably enriched in immune response by biological processes, receptor activity 208 by molecular functions, and plasma membrane by cellular component, respectively (Figure 3A209 C). Similarly, stromal up-regulated DEGs mainly enriched in cell adhesion by biological 210 processes, extracellular matrix binding by molecular functions, and extracellular space by 211 cellular component, respectively (Supplementary Figure 1A-C). KEGG pathway were both 212 mainly enriched in cytokine-cytokine receptor interaction and cell adhesion molecules (CAMs) 213 pathway(Figure 3D, Supplementary Figure 1D).

214

\section{Overall survival analysis of DEGs}

216 To make further efforts to elucidate if up-regulated DEGs could give benefits to the LUAD 217 patient survival, Kaplan-Meier survival and univariate Cox proportional hazards regression 218 analyses were performed on the up-regulated DEGs. The cases whose overall survival $<30$ days 219 were excluded. The results showed that 446 immune related DEGs correlated with patient 220 survival $(\mathrm{p}<0.05), 387$ stromal related DEGs correlated with patient $\operatorname{survival}(\mathrm{p}<0.05)$. Among all 221 those genes, there were 291 duplicates of immune and stromal genes, a total of 542 genes 222 (prognostic DEGs) associated with overall survival in patients with LUAD (Table S1).

223

\section{Construction of PPI network by prognostic gene}

Based on the STRING database, PPI network was obtained by using Cytoscape software to clarify the interaction between prognostic DEGs. The network was constructed by 13 modules, and we choose the top three significant modules ranked by mcode score for further study (Figure 4). A total of 527 nodes and 4661 edges were screened from the PPI network. According to the criteria above, eight nodes (PTPRC, ITGAM, LCP2, CTLA4, CD80, ITGAX, CD19, CCR5) were identified as hub genes. Consistently, these hub genes serve crucial roles in maintaining the top three modules.

\section{Prognostic gene validate and intersection analysis with PPI network}

To test whether these prognostic genes have prognostic significance in other LUAD cases. Microarray expression profile dataset GSE68465 from GEO database was downloaded and the data were subjected to prognostic gene selection. There are 176 genes among 542 prognostic genes cannot find in GSE68465 dataset because of platform differences. Of the remaining 366 genes, 106 genes were confirmed involving in LUAD patient survival. By intersection analysis the 106 prognostic genes with hub genes of PPI network, only PTPRC and CD19 were associated with overall survival in patients with LUAD.

\section{The correlation of PTPRC and CD19 expression with clinicopathological factors} Based on the study above, PTPRC and CD19 expression level were correlated with overall survival of LUAD patient, and PTPRC and CD19 high-expression group with longer survival. To explore the correlation of PTPRC and CD19 expression with clinical characteristic in LUAD 
246

247

248

249

250

251

252

253

254

255

256

257

258

259

260

261

262

263

264

265

266

267

268

269

270

271

272

273

274

275

276

277

278

279

280

281

282

283

284

285

patient, we analysis the PTPRC and CD19 expression level with TNM stage. The result indicated that the PTPRC and CD19 expression were negative correlated with the TNM stage of LUAD patient, and with the TNM stage rising, the expression of PTPRC and CD19 declined (Figure 5).

\section{PTPRC and CD19 could be an indicator in TME status}

To further elaborate the role of PTPRC and CD19 in LUAD, 517 samples from TCGA were divined into high/low expression group based on median expression. We implement GSEA in the high and low expression group of PTPRC and CD19. The result display that, in the high expression level whether PTPRC or CD19, the genes were basically enriched in immune relative activities including allograft rejection, complement and inflammatory response. In the low expression level of PTPRC, the genes were basically enriched in glycolysis, and typical tumor pathway including MYC-targets-V1 and MYC-targets-V2. As to the low expression group of CD19, genes were gathered in metabolic pathway. In C7 collection, many genes were concentrated in the high expression group of no matter PTPRC nor CD19, and both low expression group enrich few gene (Figure 6).

Further,we performed correlation analysis between PTPRC and CD19 and 22 kind of immune infiltration cell. The result reveal that 16 kinds of immune infiltration cell were related with the expression of PTPRC (Figure 7), 15 kinds associated with CD19 (Figure S 2).

\section{Construction and validation of the prognostic model}

Factor analysis was used to determine common axes (or dimensions) of patterns and structures, which were measured by a reduced set of 106 predicted genes. The first twelve factors explained about $81.808 \%$ of the variation in the 106 prognostic genes. After multivariate Cox regression analysis, five factors correlated with survival were obtained $(\mathrm{p}<0.05)$. Through the formula mentioned before, the final prognostic model combined with 106 candidate gene were constructed. Further, to test the predictive ability of the prognostic model, the microarray expression profile dataset GSE68465 was downloaded and we calculate the risk score for each patient. Based on the threshold risk score, we divided the patients into high- and low-risk group. We choose the log-rank test to identify the differences of OS between subgroups. The results showed that the overall survival of the high-risk patients is shorter than those in the low-risk ones in both TCGA dataset and GSE68465 ( $<<0.0001, \mathrm{p}=0.0002$ respectively, Figure 8A-B). Then, to estimate whether the prognostic model is predictive of relapse free survival (RFS). We divided the patients into high- and low-risk group compared with the median risk score, log-rank test indicate that the prognostic model we build could predict the RFS of LUAD. ROC curves were also applied to evaluate the sensitivity of the prognostic model,and the ROC curves showed that the AUC value of the prognostic model reached 0.649, 0.617 in TCGA dataset and GSE68465 respectively. The result indicates a substantially effective performance of the prognostic model for overall survival prediction(Figure 8C-D). 


\section{Comparison With Other Gene Expression Signatures}

287 We compared the prognostic model constructed by 106 candidate gene with 9-gene biomarker,

288

289

290

291

292

293

294

295

296

297

298

299

300

301

302

303

304

305

306

307

308

309

310

311

312

313

314

315

316

317

318

319

320

321

322

323

324 as it also use estimate score on TCGA datasets to build the prognostic model. ${ }^{27}$ GSE14814 included all 106 genes of our prognostic model and 9 genes needed to constructed the 9-gene biomarker. For GSE14814 data set, the 106-gene prognostic model achived a higher C-index (Cindex, 0.56; 95\% CI,0.48-0.64; $\mathrm{P}<0.01)$ compared with the 9-gene biomarker ( $\mathrm{C}$-index, 0.50 ; $95 \% \mathrm{CI}, 0.44-0.56 ; \mathrm{P}=0.7$ )(Figure $\mathrm{S} 3$ ).

\section{To optimize the model with clinical charateristics}

Further, univariate and multivariate Cox regression analysis have been executed between some of clinical pathological parameters and risk score of the prognostic model (Table1). The result showed that the risk assessment model was an independent prognostic factor for prognostic. As the constructed risk assessment model with great prognostic value, we intended to improve the prognostic accuracy by intergrating with LUAD clinicopathological factors. We designed a nomogram to predict the survival of LUAD patient by combing T stage, lymph nodes metastasis, recurrence and risk score (Figure 9A). The AUC of the model was achieved 0.716 for 3 year and 0.699 for 5 year (Figure 9D-E). Figure 9C-D show the nomogram calibration plots for predicting the overall survival of 3 years and 5 years of patient.

\section{Discussion}

Lung-cancer-related deaths is quite a large scale in the world which could be the most in patients who suffer cancer. And LUAD, as the most common type of lung cancer, is able to account for more than half of the morbidity and mortality of the lung cancer patient. ${ }^{28,29}$ Currently, growing evidence has suggested that TME plays a pivotal role in tumor initiation and progression, ${ }^{27,30}$ especially the recent immunocheckpoint inhibitors have noticeable effects on lung adenocarcinoma. However, patient prognosis and disease progression involved with TME related genes in LUAD have not been elucidated clearly. In the present study, 1910 genes involved in immune response and cell adhesion were identified by comparing different immune/stromal score of LUAD samples from the TCGA database. Besides, we performed survival analysis and revealed that 542 of them were associated with overall survival in LUAD patients. After performing cross validation through GSE68465, we obtained 106 TME related genes in which prominent correlation is found compared with prognosis situation. Of these survival-associated gene, 59 genes have been reported with the preliminary result. More importantly, among 106 TME related SurGenes, PTPRC and CD19 were highly interconnected nodes among PPI network and correlated with immune activity, exhibiting significant prognostic potential. 
325 Meanwhile, to explore the significance of these genes simultaneous changes for LUAD, we

326

327

328

329

330

331

332

333

334

335

336

337

338

339

340

341

342

343

344

345

346

347

348

349

350

351

352

353

354

355

356

357

358

359

360

361

362

363

364

constructed and validated a risk assessment model that predicted survival of LUAD based on 106 genes. The prognostic model achieved robust predictive ability by combing with the clinical parameter we filtrated by multivariate Cox regression analysis.Recently, we notice that there are other prognostic model build by immune gene in $\mathrm{LUAD}^{27}$, however, compared with their prognostic model, the prognostic model we build have some advantages. First of all, we constructed the model by factor analysis. Factor analysis is a kind of algorithms in biometrics. It represents a complex array of structure-analyzing procedures used to identify the interrelationships among a large set of observed variables and then, through data reduction, to group a smaller set of these variables into dimensions or factors that have common characteristics. It is a tool to reduce multidimensional data to lower dimensions while retaining most of the information ${ }^{31}$. Secondly, our prognostic model was constructed by immune gene and stromal gene rather than only included immune gene, considering the effect of stromal gene on tumor microenvironment. Thirdly, before used to constructed the model, these 106 genes have been performed univariate cox regression analysis, and all of these gene were correlated with the prognosis of LUAD. At last, our prognostic model is also predictive not only to overall survival, but also to relapse free survival.

Among 106 TME related genes to be associated with LUAD, we are especially interested in PTPRC and CD19, as in the PPI network, they are highly interconnected nodes.PTPRC is a member of the protein tyrosine phosphatase (PTP) family. PTPs are known to be signaling molecules that regulate a variety of cellular processes including cell growth, differentiation, mitosis, and oncogenic transformation. PTPRC has been shown to be an essential regulator of Tand B-cell antigen receptor signaling. It functions through either direct interaction with components of the antigen receptor complexes, or by activating various Src family kinases required for the antigen receptor signaling. PTPRC also suppresses JAK kinases, and thus functions as a regulator of cytokine receptor signaling. Alternatively spliced transcripts variants of this gene, which encode distinct isoforms, have been reported. There were studies showed the presence of intraepithelial PTPRC + cells from NSCLC or SCLC patients predicts favourable disease-specific survival. ${ }^{32,33}$ However, the mechanism of PTPRC in lung cancer has not been elucidated. The GSEA enrichment analysis in our research showed the genes in PTPRC highexpression group were mainly enriched in immune-related activities, such as allograft rejection, complement, and interferon response, and enriched in metabolic pathways in low expression of them, including glycolysis, oxidative phosphorylation, and typical tumor pathways. These result indicate that the expression level of PTPRC might correlate with the status of immune and microenviroment in tumor. Further study analysised by CIBERSORT algorithm showed many tumor infiltrating immune cell were correlated with the expression of PTPRC. All of these result suggest PTPRC participating in tumor microenvironment, and could be an indicator of TNM status. CD19 functions as coreceptor for the B-cell antigen receptor complex (BCR) on Blymphocytes. Decreases the threshold for activation of downstream signaling pathways and for triggering B-cell responses to antigens. ${ }^{34-36}$ Required for normal B cell differentiation and

Peer) reviewing PDF | (2019:12:44416:2:0:NEW 27 Oct 2020) 
365

366

367

368

369

370

371

372

373

374

375

376

377

378

379

380

381

382

383

384

385

386

387

388

389

390

391

392

393

394

395

396

397

398

399

400

401

proliferation in response to antigen challenges. ${ }^{35,37}$ Some studies had reported that CD19 might serve as a downstream effector in RET signaling pathway and EGFR-activated signals in NSCLC. Other research have found in subcutaneous tumor of mice injected with LP07 adenocarcinoma cells and then treated with CD19 monoclonal antibodies, compared to nontreated cancer mice, in tumors of monoclonal-treated animals, tumor area and weight and ki-67 were significantly reduced, which implied that CD19 seemed to play a protumoral role in LUAD. There were researches reported the bronchoalveolar lavage fluid subset composition in primary lung neoplasia differs from that in the peripheral blood through an increase in CD19+ B cells compared with the peripheral blood. Similarly, some studies have described CD19+ B cells as the pathogenic populations within lung tumors (Tumor-infiltrating B cells: their role and application in anti-tumor immunity in lung cancer, Bal $\mathrm{T}$ lymphocyte subsets are reduced in primary lung neoplasias) ${ }^{38,39}$ However, Our results suggested that the expression of CD19 was decreased in the advancing stages of LUAD patients. According to another study, in non small cell lung cancer, CD19 + B cells decrease by cancer disease(Biomarkers related to immunosenescence: relationships with therapy and survival in lung cancer patients). ${ }^{40}$ Thus, CD19 might play a double-face role in tumor, in different stages of tumor, either promoting survival or inducing apoptosis. The GSEA results showed that immune-related signaling pathways, such as allograft rejection, complement, and interferon response, were significantly enriched in the CD19 high expression group. In the CD19 low-expression group, metabolic pathways including adipogenesis, cholesterol homeostasis, and typical tumor pathways were enriched. These results implied that CD19 might participate in the status conversion of TME from immune-dominant to metabolic-dominant. Further analysis of TIC supported this view. Accordingly, the downregulation of CD19 along with the advancing stage of LUAD, the conversion of TME from immune-predominant to metabolic-dominant status, and the reduction of antitumor TICs supported that CD19 might play an antitumor role in LUAD.

\section{Conclusions}

In summary, after analysis of immune/stromal score by using the ESTIMATE algorithm in TCGA database, a series of TME related genes was obtained and validated by an independent LUAD cohort. Further, the prognostic model combined with those identified genes was constructed and proved could provide a moderate OS prediction for LUAD. By integrating with clinical characteristics, testing by multivariate Cox regression analysis, the predictive ability have been optimized. Among 106 TME related genes, PTPRC and CD19 are highly interconnected nodes in the PPI network. Meanwhile, bioinformatic evidence suggest the levels of them affected the immune activity of TME. These two prognostic genes might be a candidate target to monitor and treat lung cancer, attributing to their immune nature and prognostic significance, thereby improving the clinical outcomes. Finally, our study might provide a novel 
402 insight on the potential correlation between monitoring and manipulating the TME with LUAD

403 prognosis and precision immunotherapies.

404

\section{Acknowledgements}

406 Not applicable.

407

408

\section{References}

409 1. Chen W, Zheng R, Baade PD, Zhang S, Zeng H, Bray F, Jemal A, Yu XQ, He J. Cancer 410 statistics in China, 2015. CA Cancer J Clin. 2016; 66: 115-132.

411 2. Losanno T, Gridelli C. Safety profiles of first-line therapies for metastatic non-squamous non412 small-cell lung cancer. Expert Opin Drug Saf. 2016; 15: 837-851.

413 3. Nakamura H, Saji H. Worldwide trend of increasing primary adenocarcinoma of the lung. 414 Surg Today. 2014; 44: 1004-1012.

415 4. Siegel RL, Miller KD, Jemal A. Cancer statistics, 2015. CA Cancer J Clin. 2015; 65:5-29.

416 5. William WN Jr, Lin HY, Lee JJ, Lippman SM, Roth JA, Kim ES. Revisiting stage IIIB and IV 417 non-small cell lung cancer: analysis of the surveillance, epidemiology, and end results data. 418 Chest. 2009; 136:701-709.

419 6. Shaw AT, Yeap BY, Solomon BJ, Riely GJ, Gainor J, Engelman JA, Shapiro GI, Costa DB, 420 Ou SH, Butaney M, Salgia R, Maki RG, Varella-Garcia M, Doebele RC, Bang YJ, Kulig K, 421 Selaru P, Tang Y, Wilner KD, Kwak EL, Clark JW, Iafrate AJ, Camidge DR. Effect of crizotinib 422 on overall survival in patients with advanced non-small-cell lung cancer harbouring ALK gene 423 rearrangement: a retrospective analysis. Lancet Oncol. 2011; 12:1004-1012.

424 7. Cui Y, Liu J, Yin HB, Liu YF, Liu JH. Fibulin-1 functions as a prognostic factor in lung 425 adenocarcinoma. Jpn J Clin Oncol. 2015; 45:854-859.

426 8. Zhan J, Wang P, Niu M, Wang Y, Zhu X, Guo Y, Zhang H. High expression of transcriptional 427 factor HoxB9 predicts poor prognosis in patients with lung adenocarcinoma. Histopathology. 428 2015; 66:955-965.

429 9. Kai Zhao, Zulei Li, Hui Tian. Twenty-gene-based prognostic model predicts lung 430 adenocarcinoma survival. Onco Targets Ther. 2018; 11: 3415-3424. 
431 10. Wood SL, Pernemalm M, Crosbie PA, Whetton AD. The role of the tumor-

432 microenvironment in lung cancer-metastasis and its relationship to potential therapeutic targets.

433 Cancer Treat Rev. 2014; 40:558-566.

434 11. Li B, Cui Y, Diehn M, Li R. Development and Validation of an Individualized Immune

435 Prognostic Signature in Early-Stage Nonsquamous Non-Small Cell Lung Cancer. JAMA Oncol. $436 \quad 2017 ; 3: 1529-1537$.

437 12. Meyers DE, Bryan PM, Banerji S, Morris DG. Targeting the PD-1/PD-L1 axis for the 438 treatment of non-small-cell lung cancer. Curr Oncol. 2018; 25:324-334.

439 13. Liu SY, Wu YL. Ongoing clinical trials of PD-1 and PD-L1 inhibitors for lung cancer in 440 China. J Hematol Oncol. 2017; 10:136.

441 14. Mansuet-Lupo A, Alifano M, Pécuchet N, Biton J, Becht E, Goc J, Germain C, Ouakrim H, 442 Régnard JF, Cremer I, Laurent-Puig P, Dieu-Nosjean MC, Blons H, Damotte D. Intratumoral 443 Immune Cell Densities Are Associated with Lung Adenocarcinoma Gene Alterations. Am J 444 Respir Crit Care Med. 2016; 194:1403-1412.

445 15. Driver BR, Ross A Miller, Tara Miller, Michael Deavers, Blythe Gorman, Dina 446 Mody, Yimin Ge, Roberto Barrios, Eric Bernicker, Min Kim, Philip T Cagle. Programmed Death 447 Ligand-1 (PD-L1) Expression in Either Tumor Cells or Tumor-Infiltrating Immune Cells 448 Correlates with Solid and High-Grade Lung Adenocarcinomas. Arch Pathol Lab Med. 2017; 449 141:1529-1532.

450 16. Connolly KA, Nader M, Joshi N. Investigating Tumor-Associated Tertiary Lymphoid 451 Structures in Murine Lung Adenocarcinoma. Methods Mol Biol. 2018; 1845:259-273.

452 17. Yoshihara K, Maria Shahmoradgoli, Emmanuel Martínez, Rahulsimham Vegesna, Hoon 453 Kim, Wandaliz Torres-Garcia, Victor Treviño, Hui Shen, Peter W Laird, Douglas A 454 Levine, Scott L Carter, Gad Getz, Katherine Stemke-Hale, Gordon B Mills, Roel G W Verhaak. 455 Inferring tumour purity and stromal and immune cell admixture from expression data. Nat 456 Commun. 2013; 4:2612.

457 18. Shah N, Ping Wang, John Wongvipat, Wouter R Karthaus, Wassim Abida, Joshua 458 Armenia, Shira Rockowitz, Yotam Drier, Bradley E Bernstein, Henry W Long, Matthew L 459 Freedman, Vivek K Arora, Deyou Zheng, Charles L Sawyers. Regulation of the glucocorticoid 460 receptor via a BET-dependent enhancer drives antiandrogen resistance in prostate cancer. Elife. $4612017 ; 6: 27861$.

462 19. Priedigkeit N, Watters RJ, Lucas PC, Basudan A, Bhargava R, Horne W, Kolls JK, Fang Z, 463 Rosenzweig MQ, Brufsky AM, Weiss KR, Oesterreich S, Lee AV. Exome-capture RNA 
464 sequencing of decade-old breast cancers and matched decalcified bone metastases. JCI Insight. 465 2017;2: 95703.

466 20. M Henar Alonso, Susanna Aussó, Adriana Lopez-Doriga, David Cordero, Elisabet 467 Guinó, Xavier Solé, Mercè Barenys, Javier de Oca, Gabriel Capella, Ramón Salazar, Rebeca 468 Sanz-Pamplona, Victor Moreno. Comprehensive analysis of copy number aberrations in 469 microsatellite stable colon cancer in view of stromal component. Br J Cancer. 2017; 117:421470431.

471 21. Director's Challenge Consortium for the Molecular Classification of Lung 472 Adenocarcinoma; Kerby Shedden, Jeremy M G Taylor, Steven A Enkemann, Ming-Sound 473 Tsao, Timothy J Yeatman, William L Gerald, Steven Eschrich, Igor Jurisica, Thomas J 474 Giordano, David E Misek, Andrew C Chang, Chang Qi Zhu, Daniel Strumpf, Samir 475 Hanash, Frances A Shepherd, Keyue Ding, Lesley Seymour, Katsuhiko Naoki, Nathan 476 Pennell, Barbara Weir, Roel Verhaak, Christine Ladd-Acosta, Todd Golub, Michael 477 Gruidl, Anupama Sharma, Janos Szoke, Maureen Zakowski, Valerie Rusch, Mark Kris, Agnes 478 Viale, Noriko Motoi, William Travis, Barbara Conley, Venkatraman E Seshan, Matthew 479 Meyerson, Rork Kuick, Kevin K Dobbin, Tracy Lively, James W Jacobson, David G Beer. Gene 480 expression-based survival prediction in lung adenocarcinoma: a multi-site, blinded validation 481 study. Nat Med. 2008; 14(8):822-7.

482 22. Eliane Marti 1, David W Horohov, Doug F Antzak, Sandor Lazary, D Paul Lunn. Advances 483 in equine immunology: Havemeyer workshop reports from Santa Fe, New Mexico, and 484 Hortobagy, Hungary. Vet Immunol Immunopathol. 2003; 91(3-4):233-43.

485 23. Matthew E Ritchie, Belinda Phipson, Di Wu, Yifang Hu, Charity W Law, Wei Shi, Gordon 486 K Smyth. Limma powers differential expression analyses for RNA-sequencing and microarray 487 studies. Nucleic Acids Res. 2015; 43: e47.

488 24. Metsalu T, Vilo J. ClustVis: a web tool for visualizing clustering of multivariate data using 489 Principal Component Analysis and heatmap. Nucleic Acids Res.2015; 43: W566-70.

490 25. Huang W, Sherman BT, Lempicki RA. Systematic and integrative analysis of large gene 491 lists using DAVID bioinformatics resources. Nat Protoc. 2009; 4:44-57.

492 26. Bader GD, Hogue CW. An automated method for finding molecular complexes in large 493 protein interaction networks. BMC Bioinformatics. 2003; 4:2.

494 27. Genwen Chen, Zhongyi Dong, Dehua Wu, Yuhan Chen. Profiles of immune infiltration in 495 lung adenocarcinoma and their clinical significant: A gene-expression-based retrospective study.

496 J Cell Biochem. 2020;121(11):4431-4439. 
497 28. William D Travis, Elisabeth Brambilla, Masayuki Noguchi, Andrew G Nicholson, Kim 498 Geisinger, Yasushi Yatabe, Charles A Powell, David Beer, Greg Riely, Kavita Garg, John H M 499 Austin, Valerie W Rusch, Fred R Hirsch, James Jett, Pan-Chyr Yang, Michael Gould, American 500 Thoracic Society. International Association for the Study of Lung Cancer/American Thoracic

501 Society/European Respiratory Society: international multidisciplinary classification of lung 502 adenocarcinoma: executive summary. Proc Am Thorac Soc. 2011; 8:381-385.

503 29. Jemal A, Bray F, Center MM, Ferlay J, Ward E, Forman D. Global cancer statistics. CA 504 Cancer J Clin. 2011; 61:69-90.

505 30. Liotta LA, Kohn EC. The microenvironment of the tumour-host interface. Nature. 2001; $506 \quad 411: 375-9$.

507 31. Barber DF, Faure M, Long EO. LFA-1 contributes an early signal for NK cell cytotoxicity. J 508 Immunol. 2004; 173:3653-3659.

509 32. Thomas K Kilvaer, Erna-Elise Paulsen, Mehrdad R Khanehkenari, Samer Al-Saad, Ramona 510 M Johansen, Khalid Al-Shibli, Roy M Bremnes, Lill-Tove Busund, Tom Donnem. The presence 511 of intraepithelial CD45RO+ cells in resected lymph nodes with metastases from NSCLC patients 512 is an independent predictor of disease-specific survival. Br J Cancer. 2016;114(10):1145-51.

513 33. Wei Wang, Philip Hodkinson, Fiona McLaren, Melanie J Mackean, Linda Williams, Sarah E 514 M Howie, William A H Wallace, Tariq Sethi.Histologic assessment of tumor-associated $515 \mathrm{CD} 45(+)$ cell numbers is an independent predictor of prognosis in small cell lung 516 cancer.Chest 2013;143(1):146-151.

517 34. M A de Rie, T N Schumacher, G M van Schijndel, R A van Lier, F Miedema. Regulatory 518 role of CD19 molecules in B-cell activation and differentiation. Cell Immunol 1989;118(2):36851981.

520 35. R H Carter, D T Fearon. CD19: lowering the threshold for antigen receptor stimulation of B 521 lymphocytes. Science 1992;256(5053):105-7.

522 36. Menno C van Zelm, Ismail Reisli, Mirjam van der Burg, Diana Castaño, Carel J M van 523 Noesel, Maarten J D van Tol, Cristina Woellner, Bodo Grimbacher, Pablo J Patiño, Jacques J M 524 van Dongen, José L Franco. An antibody-deficiency syndrome due to mutations in the CD19 525 gene. N Engl J Med. 2006;354(18):1901-12.

526 37. M A de Rie, T N Schumacher, G M van Schijndel, R A van Lier, F Miedema. Regulatory 527 role of CD19 molecules in B-cell activation and differentiation. Cell Immunol1989;118(2):36852881. 
529 38. Si-Si Wang, Wei Liu, Dalam Ly, Hao Xu 1, Limei Qu, Li Zhang. Tumor-infiltrating B cells:

530 their role and application in anti-tumor immunity in lung cancer.Cell Mol

531 Immunol. 2019;16(1):6-18.

532 39. G Cascio, A Anania, R Mazzetti, E Scappaticci, R M Canavera, G Marinuzzi. Bal T

533 lymphocyte subsets are reduced in primary lung neoplasias. Panminerva Med.1993;35(3):127-

53430.

535 40. Danay Saavedra, Beatriz García, Patricia Lorenzo-Luaces, Amnely González, Xitlally

536 Popa, Karla P Fuentes, Zaima Mazorra, Tania Crombet, Elia Neninger, Agustin Lage.

537 Biomarkers related to immunosenescence: relationships with therapy and survival in lung cancer

538 patients. Cancer Immunol Immunother. 2016 Jan;65(1):37-45.

539 


\section{Table $\mathbf{1}$ (on next page)}

The univariate and multivariate Cox regression analysis of the patients from The Cancer Genome Atlas (TCGA) 


\begin{tabular}{|c|c|c|c|c|c|c|}
\hline \multirow[t]{2}{*}{ Parameters } & \multicolumn{3}{|c|}{ Univariate } & \multicolumn{3}{|c|}{ Multivariate } \\
\hline & HR & $95 \% \mathrm{Cl}$ & $\begin{array}{l}\text { P- } \\
\text { value }\end{array}$ & HR & $95 \% \mathrm{Cl}$ & P-value \\
\hline Recurrence(yes VS no) & 2.405 & $1.709-3.385$ & $<0.001$ & 2.449 & $1.738-3.450$ & $<0.001$ \\
\hline Age $(\leq 65 y$ VS >65y) & 1.157 & $0.860-1.558$ & 0.336 & & & \\
\hline Gender (male VS female) & 1.047 & $0.782-1.400$ & 0.759 & & & \\
\hline Risk score & 2.216 & $1.635-3.004$ & $<0.001$ & 1.897 & $1.329-2.709$ & $<0.001$ \\
\hline T stage & 2.455 & $1.697-3.553$ & $<0.001$ & 1.827 & $1.827-1.156$ & $<0.01$ \\
\hline N stage & 2.546 & $1.900-3.411$ & $<0.001$ & 2.139 & $1.517-3.017$ & $<0.001$ \\
\hline M stage & 1.027 & $0.747-1.414$ & 0.868 & & & \\
\hline TNM stage(I-II VS III-IV) & 2.686 & $1.973-3.658$ & $<0.001$ & 1.963 & $1.579-3.141$ & 0.155 \\
\hline Number-pack-years-smoked & 1.028 & $0.716-1.475$ & 0.881 & & & \\
\hline
\end{tabular}

1

2 


\section{Figure 1}

The relationship between Estimate/immune/stromal score and the prognosis of LUAD samples from TCGA.

(A) Estimate score was significantly associated with pathological stage $(p=0.0436)$. (B) Immune score showed predictive potential to pathological stage $(p=0.026)$. (C) Similarly, the lowest stromal score was found in the most progressive clinicopathological stage IV, however, it was not statistically significant $(p=0.145)$. (D) Immune score was significantly correlated with overall survival of LUAD samples. $(p=0.015)$. (E) Stromal score was not statistically significant correlated with overall, but the median overall survival of cases with higher stromal scores also showed longer than the patients with lower stromal scores (1830 d vs. $1293 d, p=0.0599, p=0.145)$.

A

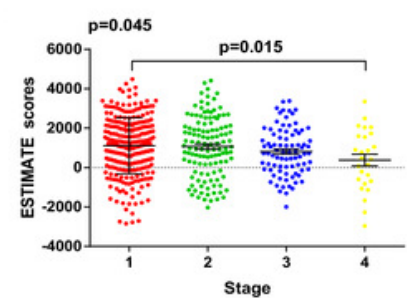

D

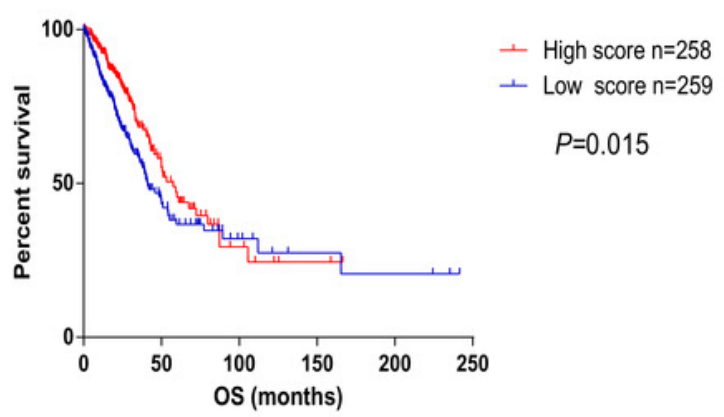

B

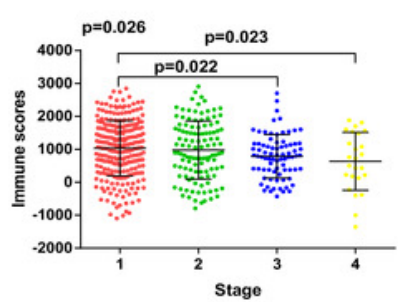

C

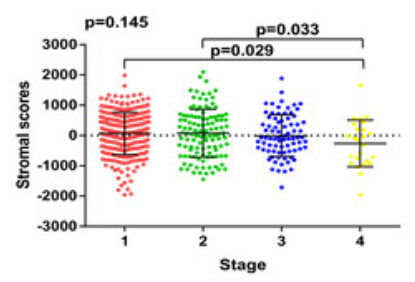
(1)

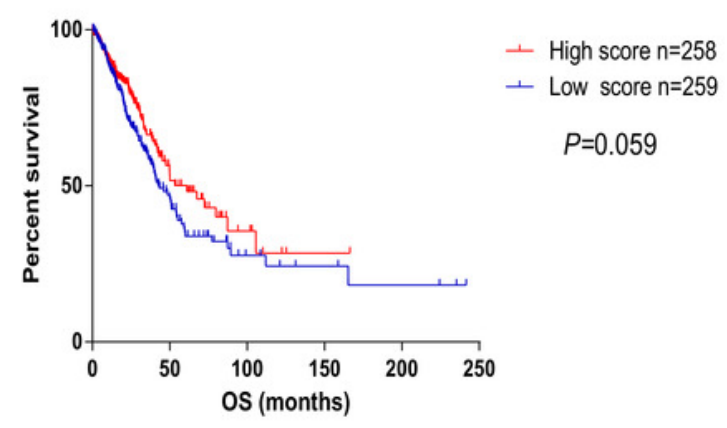




\section{Figure 2}

Differential expression genes derived from the comparision of immune/stromal high score group versus low score group.

(A-B) Heatmap of top 50 highly variaed gene from immune score /stromal score group.Genes with higher expression are shown in red, lower expression are shown in green, genes with same expression level are in black. (C-D) Venn diagram of common up /down regulated of immuneDEGs and stromal DEGs. 


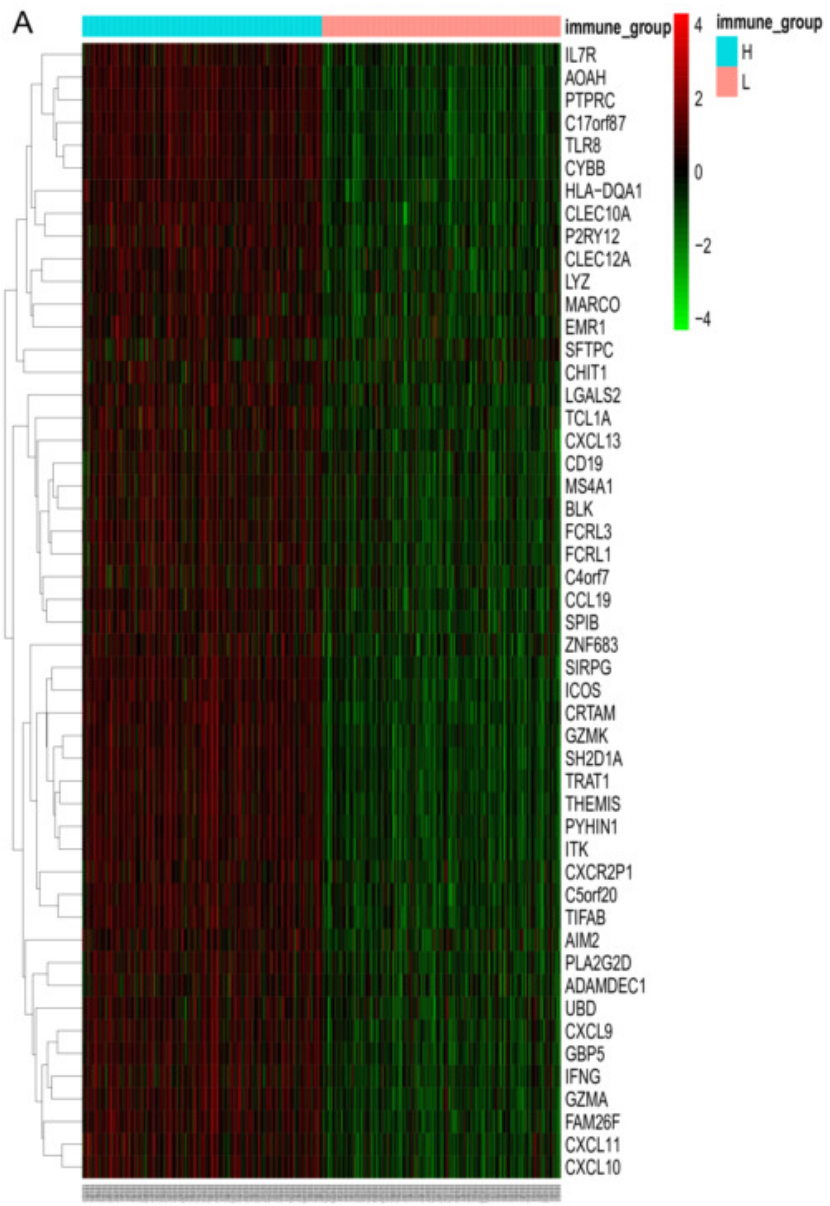

C

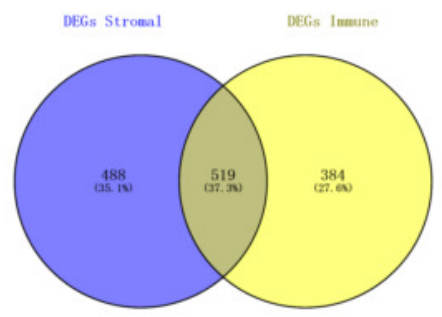

B

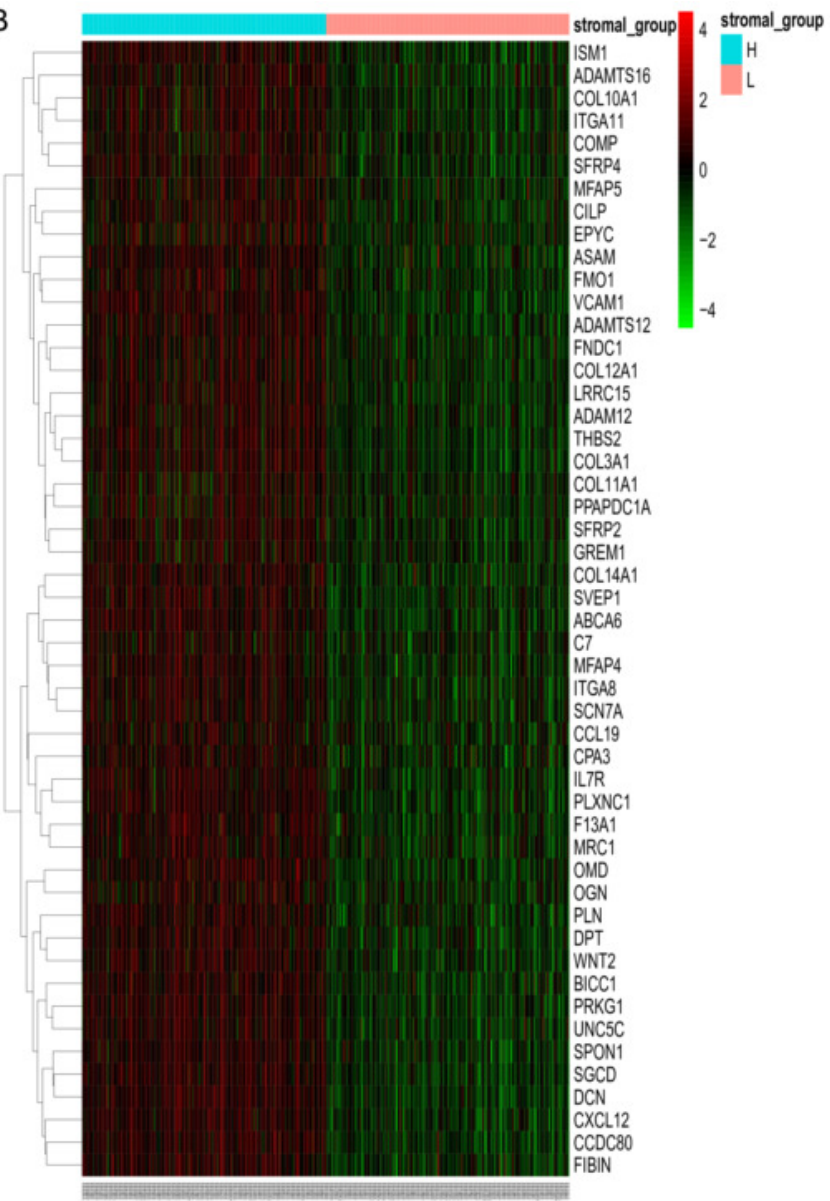

D

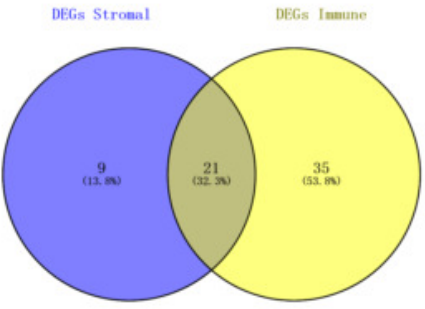


Figure 3

GO term and KEGG pathway analysis for immune up-regulated DEGs.

Top 10 pathways. False discovery rate (FDR) of GO analysis was acquired from DAVID functional annotation tool. $p<0.05$. (A) biological process, (B) cellular component, (C) molecular function, (D) KEGG pathway.

A

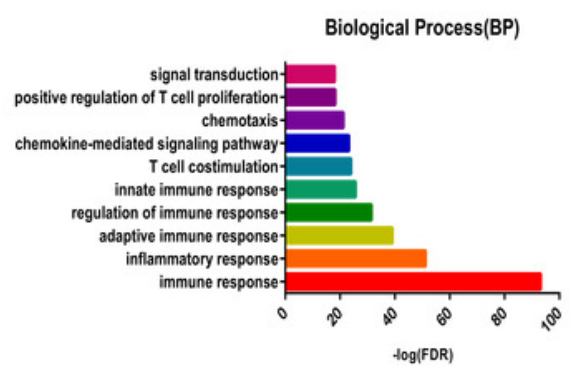

C

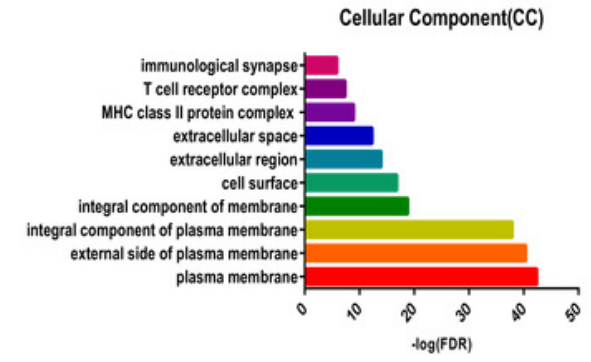

B

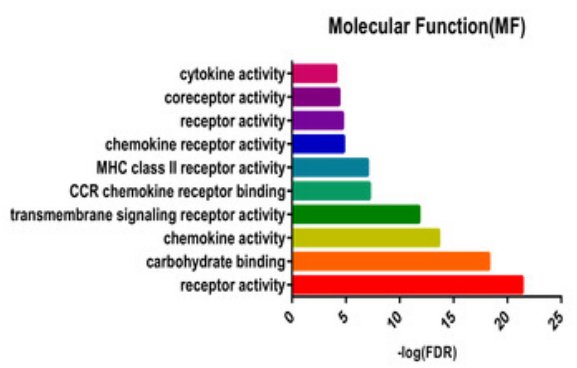

D

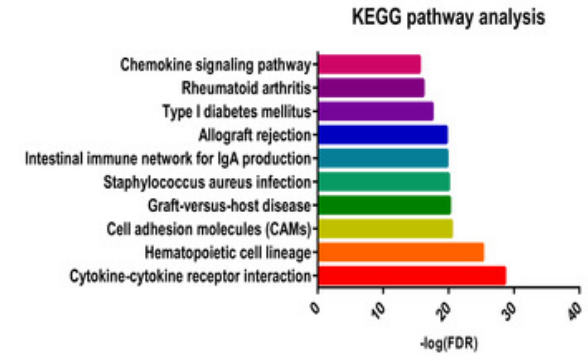


Figure 4

PPI network of top three modules, ranked by mcode score.

The color of a node in the PPI network reflects the number of interacting proteins with the designated protein, and the size of node indicates the $\log (\mathrm{FC})$ value of the $Z$ score of gene expression. 

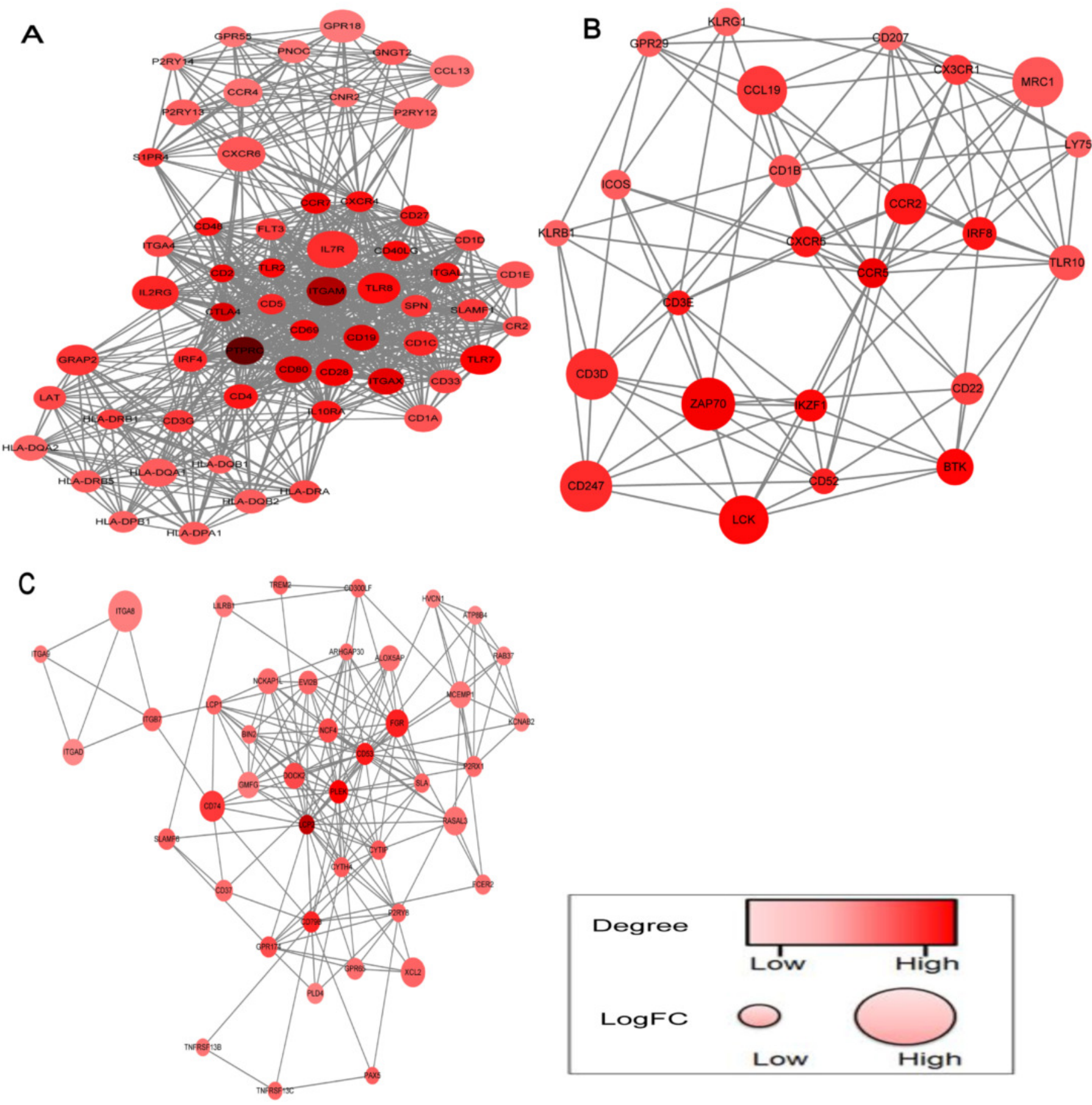
Figure 5

The correlation of PTPRC and CD19 with clinicopathological stage and survival of LUAD patients.

(A-B) The correlation of PTPRC and CD19 expression with clinicopathological stage. ANOVA served as the statistical signifificance test.

(C-D) Survival analysis for LUAD patients with different PTPRC and CD19 expression. Patients were labeled with high expression or low expression depending on the comparison with the median expression level. Logrank test served as the statistical signifificance test.

A

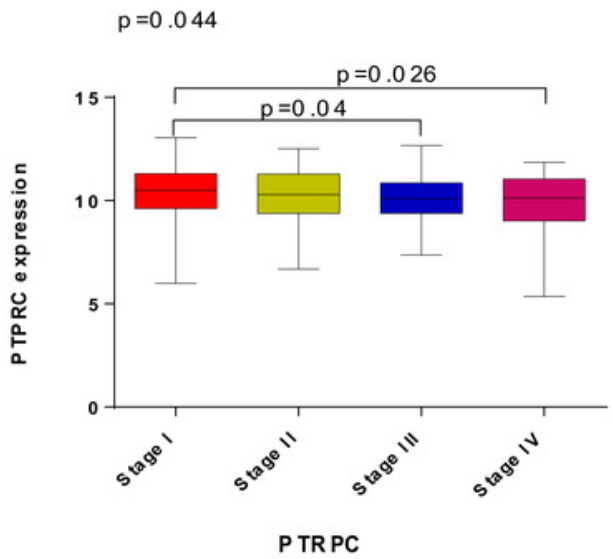

C

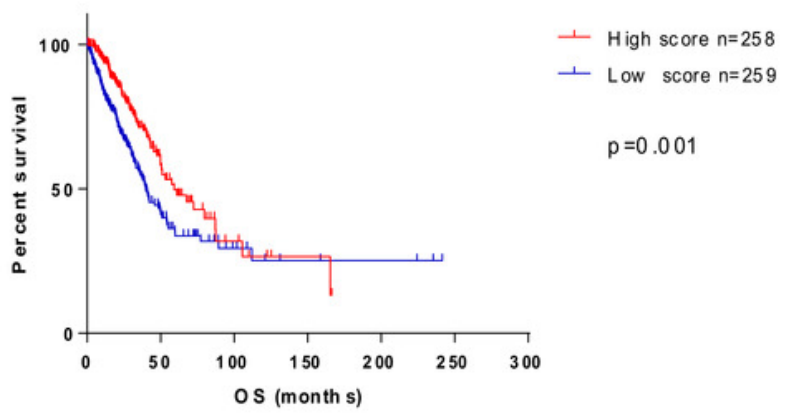

B

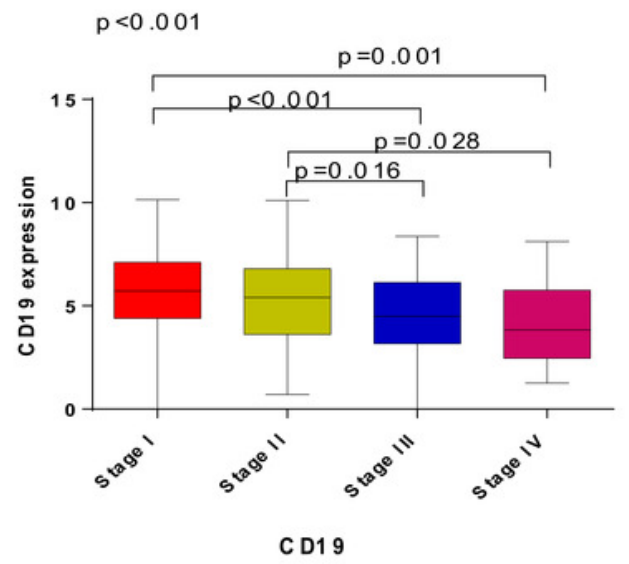

D

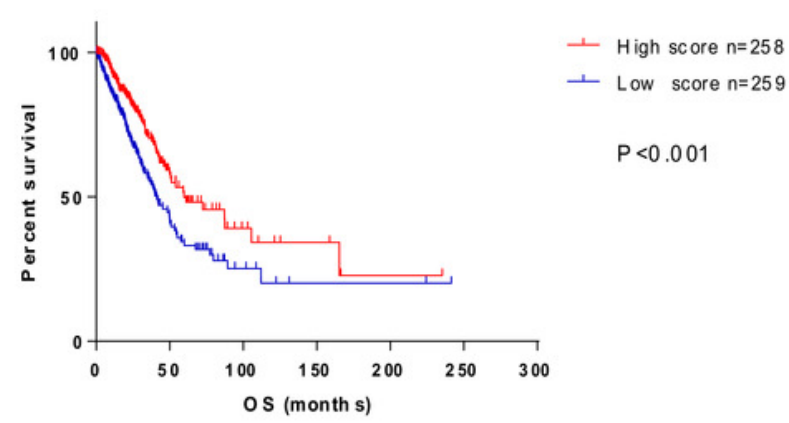




\section{Figure 6}

GSEA for samples with high PTPRC/CD19 expression and low expression.

(A-C) The enriched gene sets in HALLMARK collection by the high PTPRC expression sample. Up-regulated genes located in the left approaching the origin of the coordinates, by contrast the down-regulated lay on the right of $x$-axis. Only gene sets with NOM $p<0.05$ and FDR $q<$ 0.25 were considered signifificant. And only several leading gene sets were displayed in the plot. (D-F) The enriched gene sets in HALLMARK by samples with low PTPRC expression. (G-I) Enriched gene sets in $\mathrm{C} 7$ collection, the immunologic gene sets, by samples of high PTPRC expression. Only several leading gene sets are shown in plot. (J-K) Enriched gene sets in C7 by the low PTPRC expression. (L-N) The enriched gene sets in HALLMARK collection by the high CD19 expression sample. (O-P) The enriched gene sets in HALLMARK by samples with low CD19 expression. (Q-S) Enriched gene sets in C7 by the high CD19 expression.
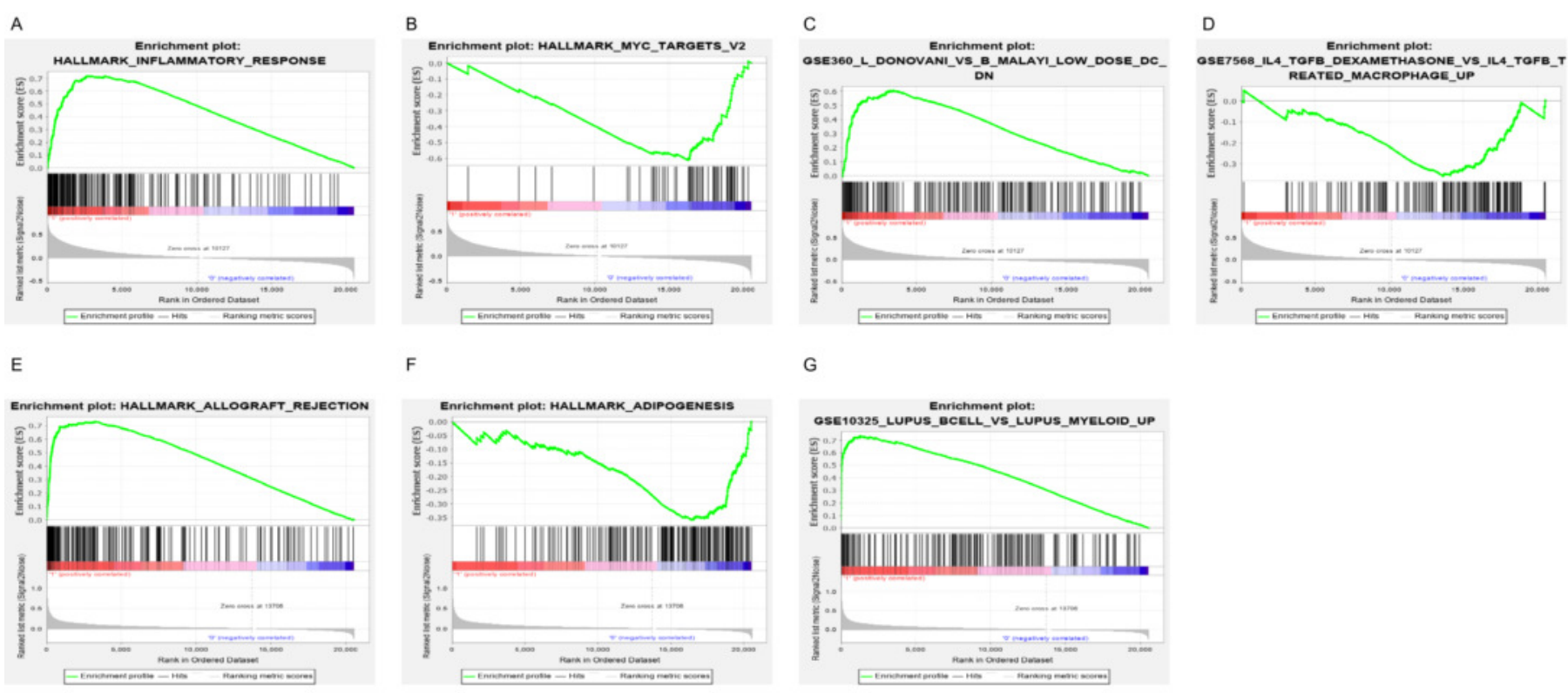

G

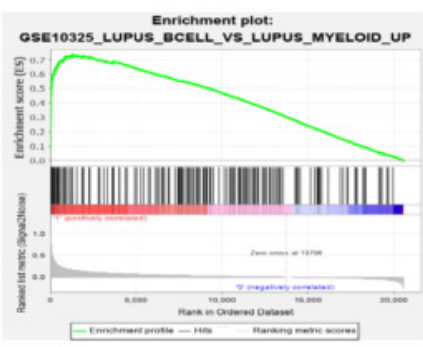




\section{Figure 7}

TIC profifile in tumor samples and correlation of TICs proportion with PTPRC expression.

(A) Barplot showing the proportion of 22 kinds of TICs in LUAD tumor samples. Column names of plot were sample ID. (B) Heatmap showing the correlation between 10 kinds of TICs and numeric in each tiny box indicating the $p$ value of correlation between two kinds of cells. The shade of each tiny color box represented corresponding correlation value between two cells, and Pearson coeffificient was used for signifificance test. (C-R) Scatter plot showed the correlation of 16 kinds of TICs proportion with the PTPRC expression $(p<0.05)$. The red line in each plot was fifitted linear model indicating the proportion tropism of the immune cell along with PTPRC expression, and Pearson coeffificient was used for the correlation test.
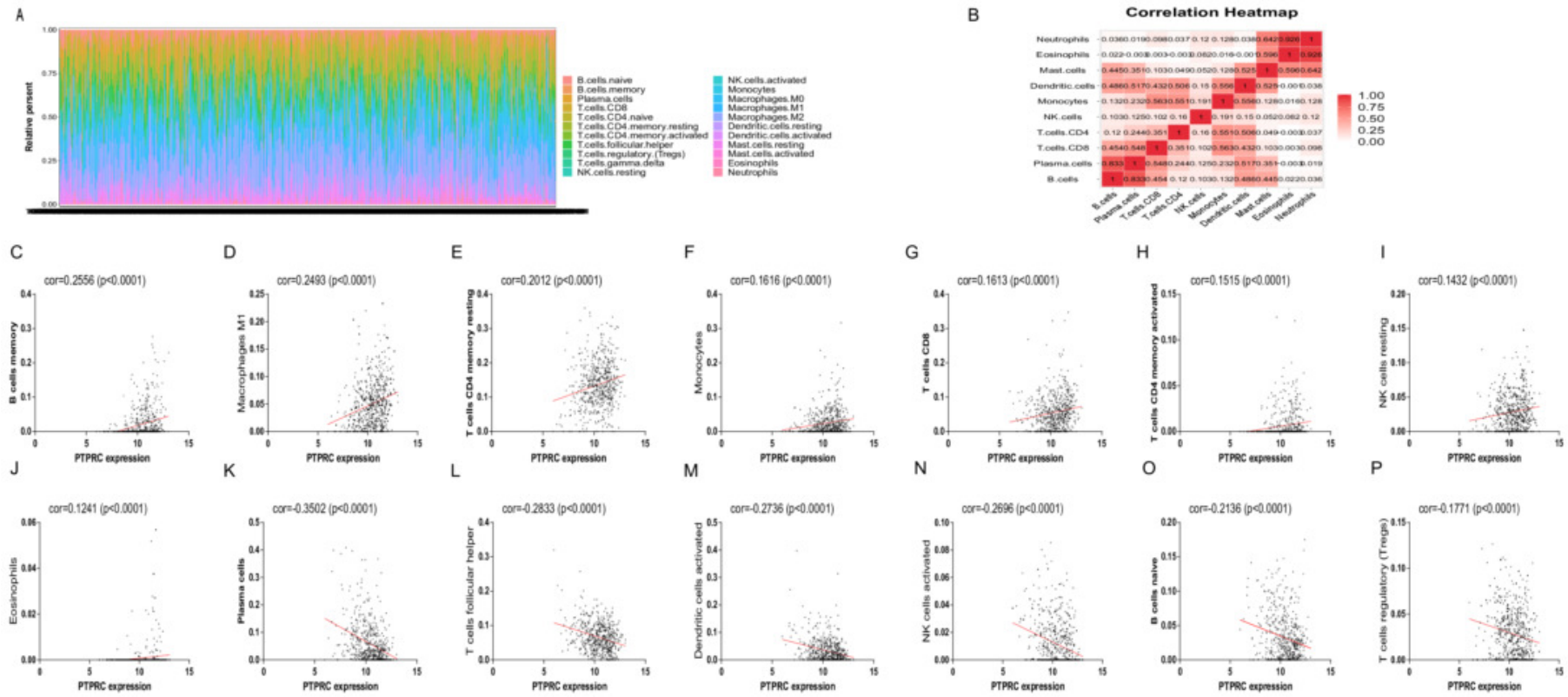


\section{Figure 8}

Validation of the prognostic model.

(A) The Kaplan-Meier survival analysis of the prognostic model for LUAD samples from TCGA. (B) ROC curve of the prognostic model in LUAD samples from TCGA. (C) The Kaplan-Meier survival analysis of the prognostic model for LUAD samples from GSE68465.(D) ROC curve of the prognostic model in LUAD samples from gse68465. (E) The Kaplan-Meier survival analysis of the prognostic model with relapse free survival for LUAD samples from TCGA. 
A

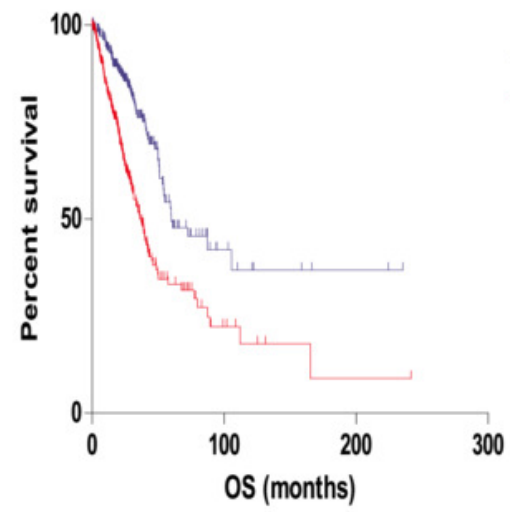

C

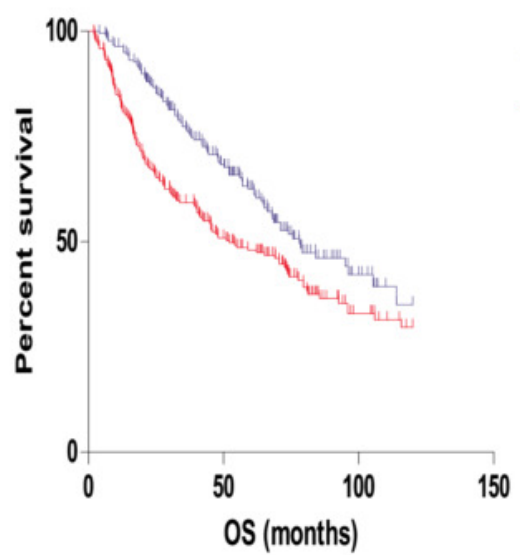

E

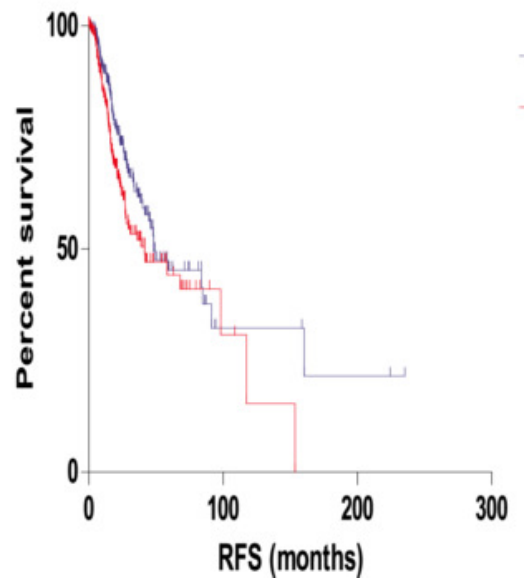

- Low risk $n=258$

- High risk $\mathrm{n}=259$

$P<0.0001$

- Low risk $\mathrm{n}=219$

$\perp$ High risk $n=220$

$P=0.0009$

- Low risk $n=214$

$\perp$ High risk $n=215$

$P=0.024$
B

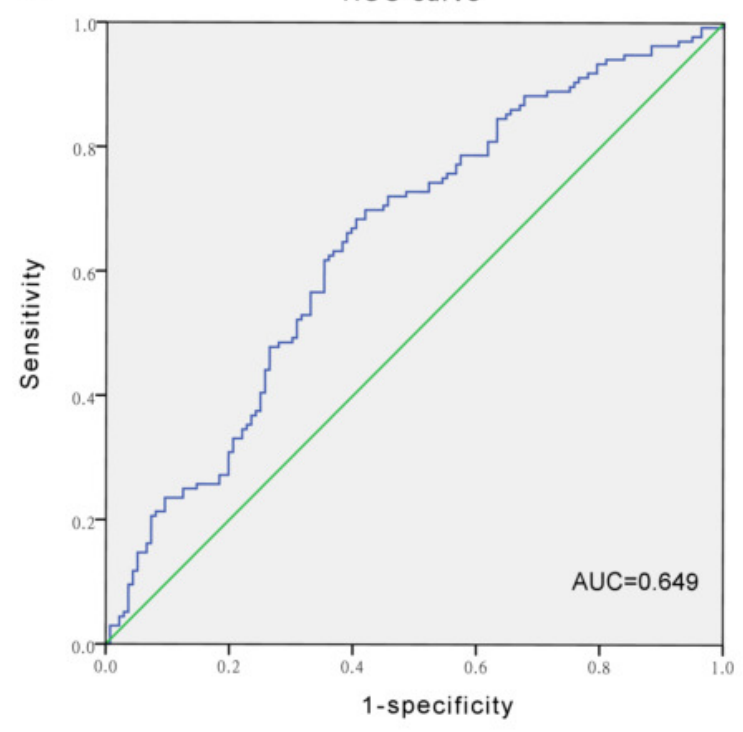

D ROC curve

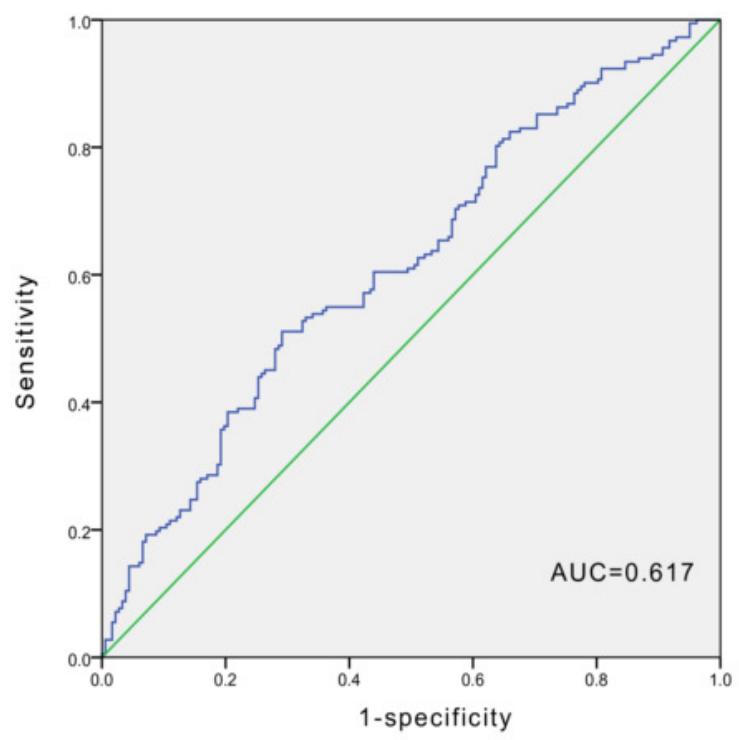


Figure 9

Nomogram and Calibration curve

$\square A$ ) Construction of a nomogram for predicting survival probability at 3,5, and year of LUAD cases from TCGA data set.

Calibration curve for the nomogram when predicting 3 (B) and 5 (C) year OS.

(D-F) ROC curve of the optimized model in 3-years and 5 years. 
A

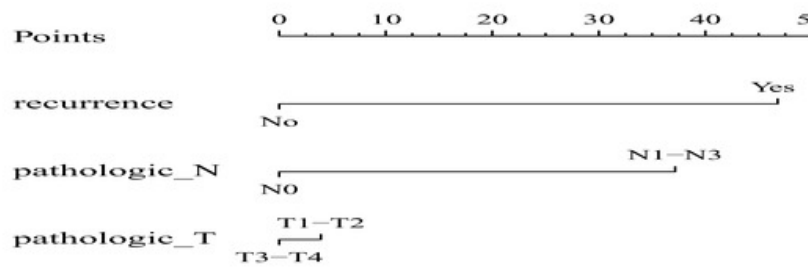

risk_score

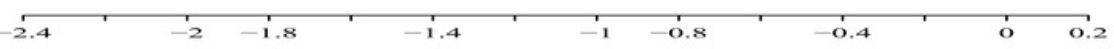

Total Points

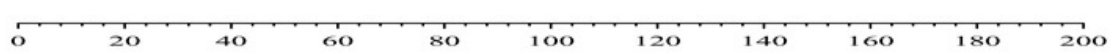

3-Year Survival

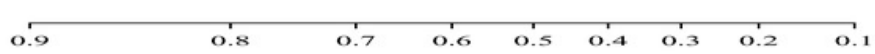

5-Year Survival

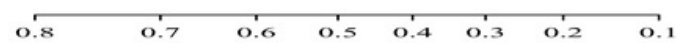

B

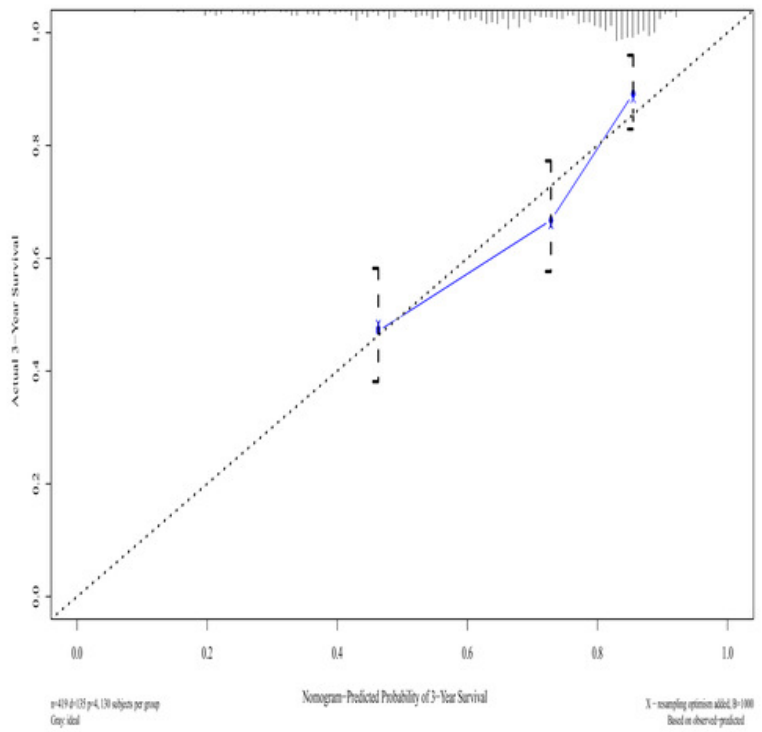

D

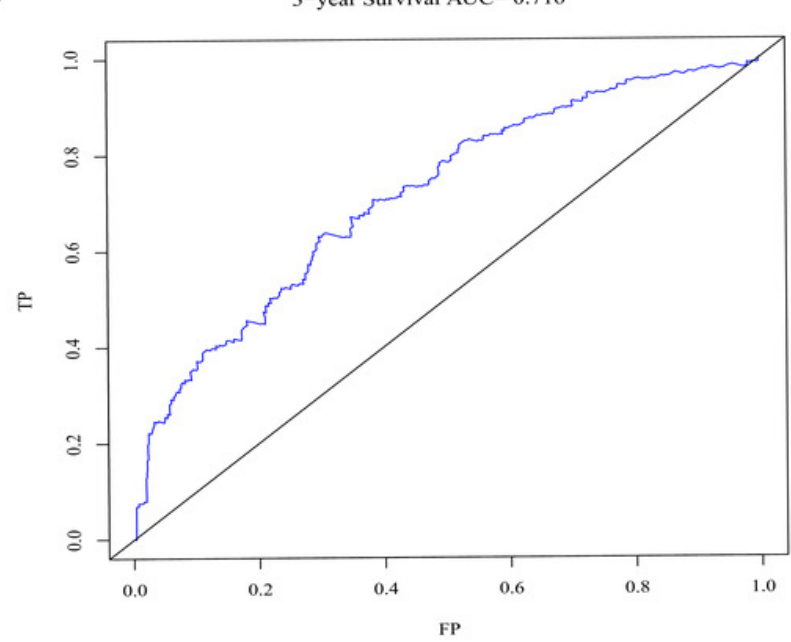

C
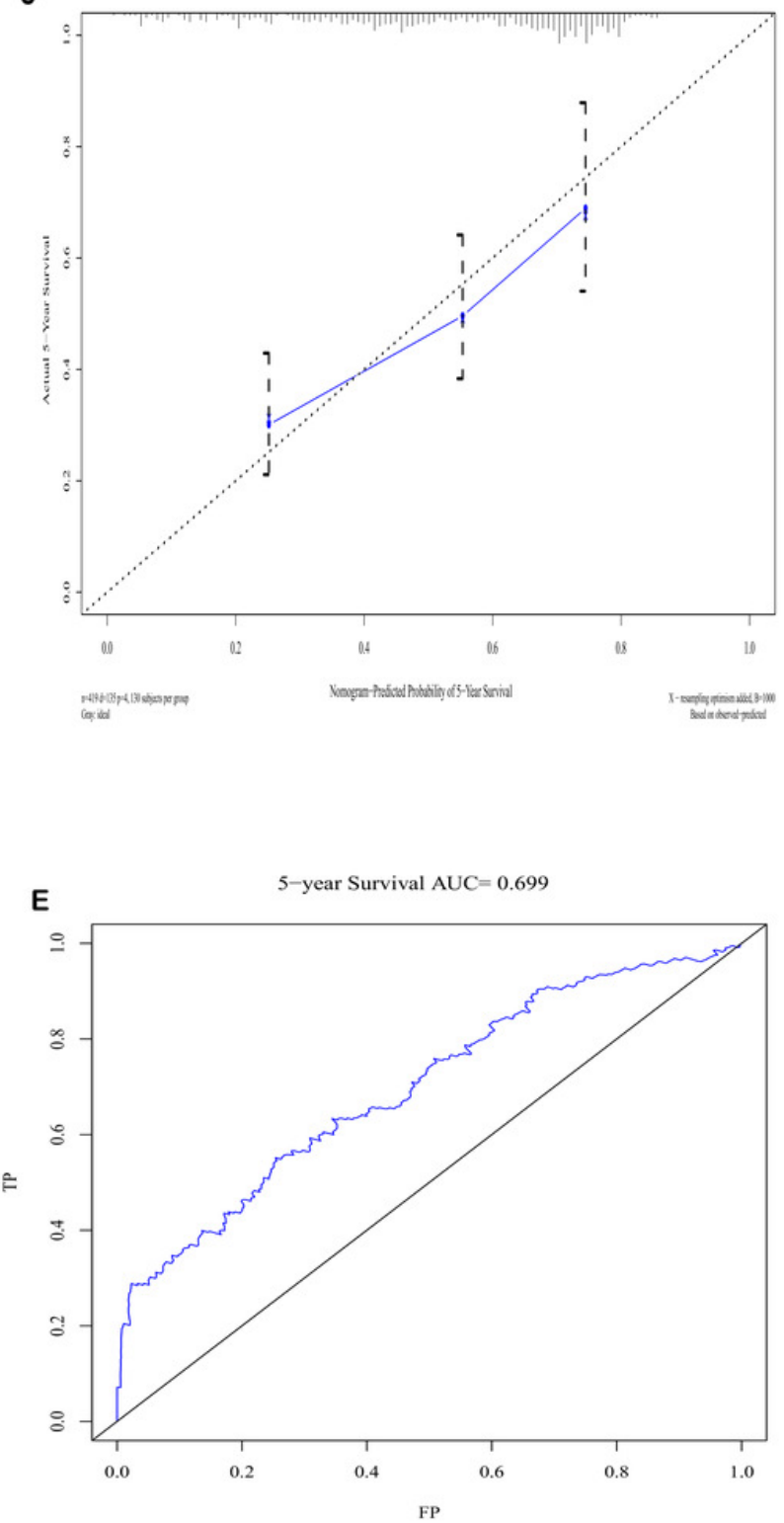